\title{
COMPUTER TECHNOLOGY \\ DADTA VI: A minicomputer-based video control system for the analysis of behavioral and electrophysiological data
}

\author{
STEVEN D. CUTCOMB, R. BRUCE BOLSTER, and KARL H. PRIBRAM \\ Neuropsychology Laboratories, Department of Psychology and Department of Psychiatry and \\ Behavioral Science, Stanford University, Stanford, California 94305
}

\begin{abstract}
A computer-automated testing apparatus for monkey and human subjects is described. This dual-computer system generates programmable color video displays and permits on-line collection of behavioral and electrophysiological data. Video displays are generated by an Apple II minicomputer linked via a serial transmission port to a general-purpose laboratory computer (DEC PDP-11/34). Control of event sequences is determined by serial interaction between the two computers. Software (programs) for the collection and analysis of event-related brain potentials data is described, and the experimental and clinical applications of this DADTA VI (Discrimination apparatus for discrete-trial analysis, Version 6) for neuropsychology are discussed.
\end{abstract}

The DADTA (discrimination apparatus for discretetrial analysis) was an automated training and testing apparatus developed during the late 1950 s and early 1960 s to investigate cognitive capacities in primates (Pribram, Gardner, Pressman, \& Bagshaw, 1962). Subsequent DADTA systems maintained the basic primate enclosure and reward-delivery design of the original DADTA system, but they evolved to incorporate laboratory minicomputers (Digital Equipment Corporation, DEC, PDP-8/e; Pribram, 1969) and black-and-white video display (Drake \& Pribram, 1976). This paper presents the newest DADTA system in its current configuration. DADTA VI incorporates the full behavioral data processing capacity of earlier versions, but a flexible color video display for stimulation is used, at no cost in CPU time. The DADTA VI system is also capable of realtime digitizing of time-locked electrophysiological data, and this capability enhances the capacity of the system for direct neuropsychological applications. The integrated hardware and software of this DADTA implementation are described below.

\section{COMPUTER LABORATORY}

The DADTA VI system depends upon the interaction of two standard laboratory minicomputers and their

The authors gratefully acknowledge the valuable assistance of Ronald Ruff in the development of the DADTA VI hardware and testing protocol. This work was supported by NSF Grant SED 78-17362 to K. H. Pribram. Requests for reprints should be addressed to Steven D. Cutcomb, Department of Psychology, Building 420, Stanford University, Stanford, California 94305 . peripherals, as well as a small amount of custom hardware interfacing (see Figure 1). Main software control of stimulus scheduling, data collection, and event timing resides in the DEC PDP-11/34a minicomputer, which runs the RT-11 (real-time) single-job operating system. Peripherals linked to this unit include a DEC VT55 console terminal, Centronix line printer, display monitor, and digital plotter. The computer is equipped with 32 kilowords $(\mathrm{Kw})$ of random-access memory (RAM) and dual high-speed magnetic disk drives (RK05), with a 3.75 megaword storage capacity. The PDP-11 interfaces to the DADTA hardware via the AR11 real-time subsystem, which has 16 analog-to-digital (A/D) conversion channels, four TTL-compatible digital output channels, two digital-to-analog output channels, and a crystalcontrolled real-time clock.

The display portion of the DADTA VI system is supported by an Apple II minicomputer, which has $24 \mathrm{Kw}$ of RAM, twin minifloppy-disk drive units, and a "GAME $1 / 0$ " port. This port is a 16-pin integratedcircuit socket with three input and four output TTLcompatible digital channels, by which serial communication with the AR11 is performed. The Apple II is used in the DADTA VI system due to its color high. resolution graphics capability, but any minicomputer with color graphics and a digital port could be used as an alternative design (cf. Durrett, 1979). Our color display is an RCA FD440 19-in. television that receives a UHF signal on Channel 33 from the Apple II, via a UHF modulator that transforms the standard video from the Apple. An unmodified video output from the Apple II can be used to drive a color monitor display, but a color monitor is much more costly than a television set, and 


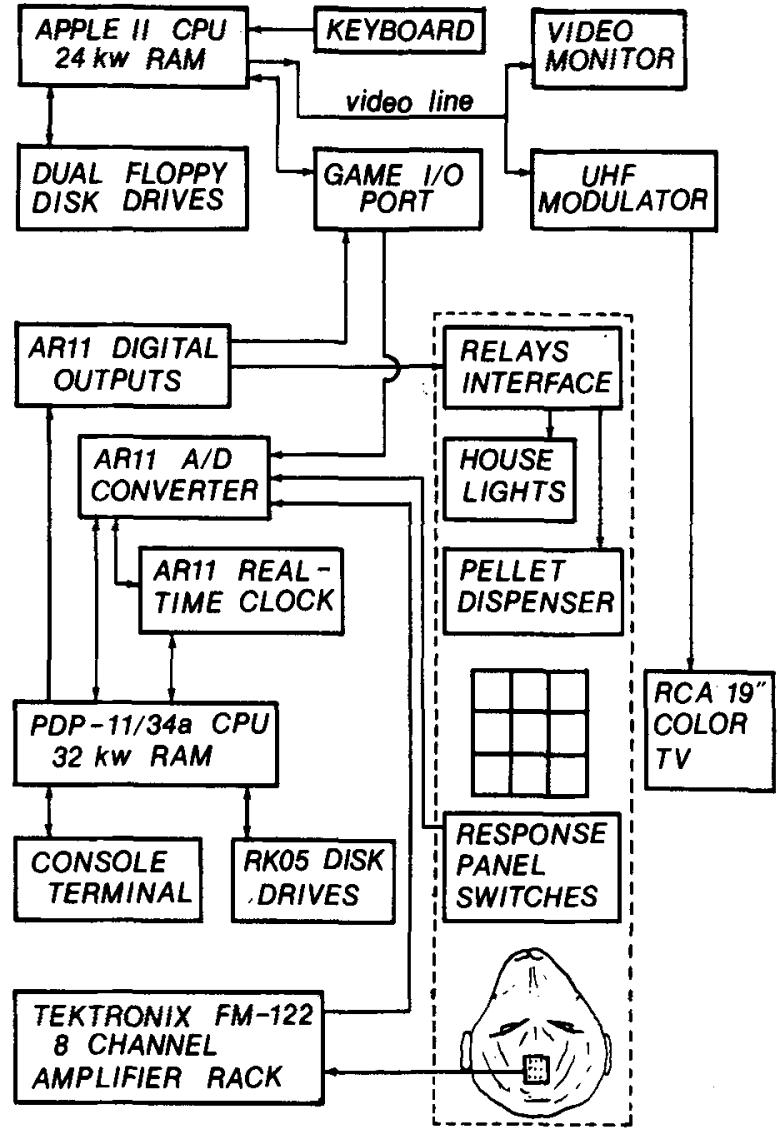

Figure 1. Diagram of the main hardware components of the DADTA VI laboratory, with arrows indicating transfer of information or control. The dotted line indicates the boundary of the testing chamber enclosure (modified refrigerator) and the devices within or attached to it.

display quality is comparable (Cavanagh \& Anstis, 1980).

\section{INTERCOMPUTER LINK}

Digital communication between the two computers in the DADTA VI system is essential. Typically, asynchronous $\mathrm{I} / \mathrm{O}$ between a CPU and a peripheral involves a buffer register for the transmitted data, as well as flag and control flip-flops for asynchronous or interrupt signaling of request to send and transmission completed (Stone, 1972, chap. 8). In the DADTA VI system, each computer uses two digital output channels that connect directly to two digital inputs at the other computer (AR11 to GAME I/O port). For each computer, the function of these two output lines is determined dynamically during the experimental session, since each program knows when to be sending and when to be receiving a transmission. When the mode is transmit, the two output lines signal data ready and data value ( 0 or 1$)$, and the two output lines from the receiving end at the other computer signal ready to receive and data received. When the role of transmitter and receiver reverses for each computer at predetermined points in the event-control sequence, the role of the lines also reverses. This technique of serial communication is very low in cost compared with a parallel or serial $\mathrm{I} / 0$ module at each computer, which would cost in excess of $\$ 650$.

\section{DADTA HARDWARE}

The DADTA enclosure and interfaces were constructed to enable noise-free computer sensing of behavioral responses and amplified electrophysiological signals. Monkey subjects are restrained in a Lucite chair inside an electrically shielded enclosure, within easy arm's reach of a 3 by 3 Lucite response-panel matrix at the rear of the enclosure. The display television is located directly behind the response panel for monkey subjects. Tc ensure artifact-free electrophysiological signals, it was necessary to insulate all metal surfaces within the enclosure from the monkey's touch. The response. detection circuitry consists of nine microswitches, one behind each Lucite panel of the 3 by 3 matrix. Wher human subjects are run, a 3 by 3 calculator keypad i: substituted for the response panel. The nine response lines are connected without buffering to nine $A / L$ channels of the AR11 device. An external power supply is used to create a $5 . \mathrm{V} 100-\mathrm{mA}$ signal at the correspond ing $A / D$ channel when a response key or panel is pressed

The relays interface consists of a shielded box with : buffer and relay for each of the two ac circuits con trolled (see Figure 1). The AR11 open-collector output ERASE L and NON-STORE are each connected with 1 -Kohm pull-up resister to $+5 \mathrm{~V}$ and then connect to thi inputs of a pair of transistors (2N697) that serve ti buffer the AR11 from the relays. These buffers connec directly to the coils of two relays, creating compute control of the pellet-dispensation and enclosure house lights. Due to inductance effects from these relays, th relays interface box was shielded and physically isolater from the main DADTA VI enclosure.

\section{DADTA VI SOFTWARE}

The operational characteristics of the DADTA V system are defined by the software that controls th event sequences of a testing session. Such a sessio consists of a series of discrete trials (usually 60-108 trials in each of which a subject is presented with a visua display, and a panel (or key) press is expected from th subject to indicate choice. Monkey subjects are rewarder for correct responses, but human subjects are not tangibl rewarded. During a testing session, behavioral data, sucl as correct trial total, panel pressed, response latency and cumulative percent correct responses, are displaye on the PDP-1 1 console, being updated after each response These discrete-trial data are saved on a disk file fo permanent storage.

The sequence of software control decisions and event that structure an individual trial is depicted in Figure 2 Prior to a testing session, the intercomputer link 


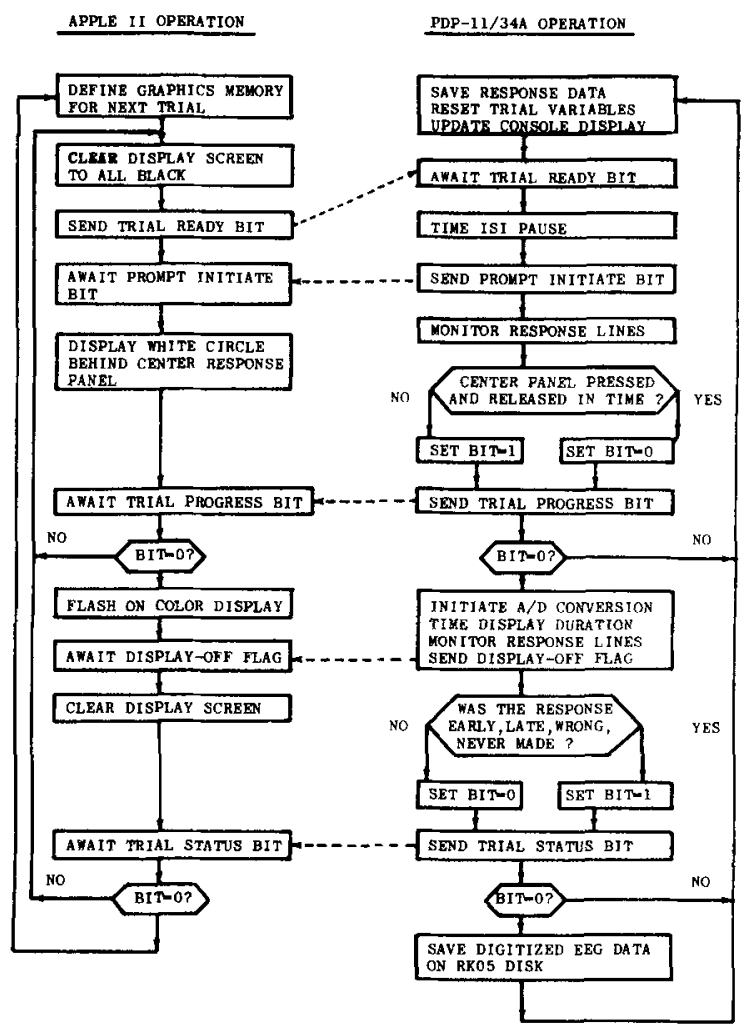

Figure 2. Flowchart of the events during a single-trial operation of a DADTA VI testing session. Solid arrows indicate control exerted by the computer over its peripherals, or decision flow within a computer. Dotted arrows indicate intercomputer communications in the flow of the Apple II and PDP-11 programs simultaneously running.

verified by software, and the PDP-11 transmits a sequence of 4-bit nybbles, each nybble being an encoded position value, from one to nine, defining the correct response panel for a trial in the testing session. After an evenparity check of the transmitted nybble sequence, the Apple II defines its graphics memory for the first highresolution graphic display, and the television screen in front of the subject is cleared to all black. In the display used in one of our protocols, there are nine individual stimulus shapes, each containing about 1,000 highresolution points. The time required for the Apple II to plot these 9,000 points/display is between 3.4 and $4 \mathrm{sec}$ and is the rate-limiting step in the event sequence of each trial. When the Apple II has defined the display memory, it transmits a bit via the serial port to the PDP-11, signifying "trial ready." The PDP-11 then times the desired intertrial interval pause and awaits a state of no panels pressed. When this state has existed for the desired time (a user-selectable parameter) the PDP-11 sends a bit to the Apple II to signal "initiate prompt." At this point, the display screen is illuminated with a single text character, 0 , which appears behind the center panel. To properly initiate the trial, the subject must respond to this prompt by pressing and releasing the center panel within timing restrictions (e.g., an error results from a press too soon or too late or for a slow panel release). Next, the PDP-11 sends a trial-progress bit to the Apple II, which signals to restart the trial if an error had been committed or to proceed with the brief visual display otherwise. The display is terminated after the desired duration (in milliseconds), when the PDP-11 sets a flag line (AR11 Line DSP CH02) high and this is sensed at the Apple II. This sensing is done by a busy-wait loop at the Apple II, reading the digital input value of GAME I/O Pin AN3. To blank the display screen, a TEXT:HOME command in APPLESOFT BASIC is executed, leaving the high-resolution graphics memory intact in case the same display is to be repeated due to an error on the present trial.

Errors may occur when any of a number of timing constraints are violated, such as responding with an incorrect panel press, multiple panel presses, or a press that is too fast or slow. The timing and response contingency parameters are set to default values at the start of each program run, but the user may alter these values interactively at run time by use of the console. If the display is presented and an error occurs, the trial is not considered completed and the same display will repeat until the desired response is made. This saves the real-time delay of defining the Apple II graphics memory (see above). Also, as an operant procedure, repeating incorrect trials until the subject makes a correct response has several advantages. Error tendencies extinguish before they develop into habits, and the number of correct presses to individual stimuli or panels may be held at the desired level to ensure balanced performance. At the end of the program run, the experimenter is presented a summary of the behavioral data at the console terminal.

The behavioral data summary is saved along with the data from each individual trial in a FORTRAN-formatted ASCII file for future reference. The behavioral data summary includes percent correct, average latency, and number of presses (total) for each panel position. This summary forms the data base to be compared with the event-related potentials data in making neuropsychological inferences.

\section{DADTA VI APPLICATIONS IN NEUROPSYCHOLOGY}

Research underway at the Stanford University Neuropsychology Laboratories entails collection of behavioral and electrophysiological data from both human and monkey subjects. The versatility provided by program control of the visual stimulus display contents, task variables, and session protocol accommodates a variety of concurrent experiments. For experiments involving electrophysiological data collection, the combined power of two computers provides advantages that are not readily accessible to either alone (e.g., Brickett, Davis, Grabert, \& Modigliani, 1980). The programs for the PDP-11 that perform on-line digitizing of electro- 
physiological data during each trial are presently designed to sample these data upon every trial but to save these time series only after the correct completion of a trial. Since program control of the video display is allocated fully to the Apple II, the high-speed FORTRAN IV and MACRO-11 programs on the PDP-11 are able to both digitize and preprocess (edit for artifact) the physiological data on each trial.

In an earlier version of the DADTA system, when bioelectric data were recorded, the data were stored as analog representations on FM tape. These data could then be analyzed off-line, but the computer-use time was effectively doubled, since the data had not been digitized on-line. In the present configuration, off-line data analysis is required only for high-level statistical processing of the data, such as sorting and averaging the raw digitized time series and subsequent feature extraction from the averages. The inclusion of a mass-storage device such as a magnetic-tape transport would permit direct data compatibility with institutional computers.

Potential users of the DADTA VI system may wish to explore the stand-alone capability of the Apple II for video control and data acquisition. The AI02 realtime analog system and the programmable real-time clock (Mountain Hardware) boards available for the Apple II can enhance a basic Apple II configuration to enable acquisition of both behavioral and electrophysiological data. At present, our twin minifloppy-disk storage system would not handle the rapid-storage demands of the digitized data processed by DADTA VI, but high-speed mass-storage devices are presently in development for the Apple computer series. A completely Apple II-based DADTA system could be used to generate and analyze behavioral data on any of the neuropsychological problems appropriate to DADTA VI. An Apple II-based system capable of full behavioral data processing would currently cost about $\$ 4,000$ for the computer hardware and $\$ 400-\$ 500$ for the color television and response-panel hardware.
DADTA has proved to be a powerful automated testing apparatus for use in primate neuropsychological investigation. The reduction of experimenter-induced bias and the ease of routine data collection using this system are critical advantages. BASIC language programming is easy on the Apple II, and the battery of testing programs developed for earlier video display systems can be readily implemented on the DADTA VI. These advantages would be equally apparent in a clinical setting, in which a system similar to DADTA VI could be used to develop a standardized neuropsychological battery.

Apple II software for DADTA VI is available on minifloppy disk, and PDP-11 software for DADTA VI is available on nine-track magnetic tape from the first author, Building 420, Jordan Hall, Stanford University, Stanford, California 94305.

\section{REFERENCES}

Brickett, P., Davis, C., Graeert, H., \& Modigliani, V. Dual on-line computers for research in cognitive psychology. Behavior Research Methods \& Instrumentation, 1980, 12, 248-250.

Cavanagh, P., \& Anstis, S. Visual psychophysics on the APPLE II: Getting started. Behavior Research Methods \& Instrumentation, 1980, 12, 614-626.

Drake, K., \& Pribram, K. H. DATA IV: A computer based video display control and data collection system for behavioral testing. In P. Brown (Ed.), Computer technology in neuroscience. New York: Wiley, 1976.

DURRETT, H. Color display systems: The state of the art. Behavior Research Methods \& Instrumentation, 1979, 11, 127-130.

Pribram, K. H. DATA III: An on-line computerized system for the experimental analysis of behavior. Perceptual and Motor Skills, 1969, 29, 599-608.

Pribram, K. H., Gardner, K., Pressman, G., \& Bagshaw, M. An automated discrimination apparatus for discrete trial analysis (DADTA). Psychological Reports, 1962, 11, 247-250.

Stone, H. Introduction to computer organization and data structures. New York: McGraw-Hill, 1972.

(Received for publication May 6, 1980; revision accepted May 27, 1981.) 